\title{
Therapy strategies regarding cytokines involvement in the pathogenesis of inflammatory bowel disease
}

\author{
Tsvetelina Velikova* \\ Clinical Immunology, University Hospital Lozenetz, Kozyak 1 str, Sofia 1407, Bulgaria
}

The choice of an appropriate therapy is one of many problems regarding management of inflammatory bowel disease (IBD) patients. However, the current therapeutic strategies include immunomodulatory drugs as 5-aminosalycilic acid agents (5-ASA), steroids, immunosuppressive and biological therapy, all directed to reduce mucosal inflammation of the patients.

In our study, "Alterations in cytokine gene expression profile in colon mucosa of Inflammatory Bowel Disease patients on different therapeutic regimens" (2018), we aimed to investigate the differences in mRNA expression of key cytokines related to Th17 and Treg cells in paired mucosal samples from IBD patients on different therapeutic regimen. We observed opposite changes in the expression of IL- 6 and TGFb1 for paired mucosa in the different groups of treated patients. We detected that IL-6, IL-10 and IL-17A expression were enhanced in the patients on 5-ASA treatment, whereas IL-23 and TGFb1 were downregulated compared to the non-treated group [1]. On the contrary, IL- 6 and IL-17A were downregulated in the immunosuppressive treated patients, simultaneously with upregulated TGFb1 and IL-10 compared to the non-treated group. Thus, by decreasing the cytokines prerequisite for Th17 developing (IL-6, TGFb1, IL-23), the immunosuppressive drugs lowered the IL-17 levels. The observation that 5-ASA treatment modulates cytokine gene expression by upregulation of IL- 6 and downregulation of TGFb1 suggests that Th17 differentiation predominates in patients with active IBD. This suggestion is supported by our results for the upregulated IL-17A expression in these patients [1]. In conclusion, we stated that treatment with immunosuppressive therapy is more beneficial for driving cytokine expression to restore immune regulation in patients with IBD.

Transcriptomics allows Investigation of cytokine gene expression profiles and developing more effective therapies to individual patients with IBD. Furthermore, obtained mucosal gene signatures in IBD patients could be fully applicable to personalized medicine approach and may predict response to treatment [2]. However, the complexity of IBD pathogenesis is well recognized including many components, i.e. environment, genetic factors, gut microbiota and mucosal immune response, each of these extremely complex on its own. Thus, the cytokines as a part of the "immunome" are not the single players in the pathogenesis. Nevertheless, the cytokine targeting approaches remain effective therapies for individual patients to provide the best possible care to IBD patients.

The advantage of cytokine inhibition would be more efficient if we know "critical cytokine," the one that is the master regulator of immune responses involved in the IBD pathogenesis. Blocking this cytokine may switch off inflammation, ideally without unwanted side effects. However, a major challenge when developing therapies that target cytokines is to not block the beneficial for the host integrity immune responses. In line with this, targeting inflammatory cells could be also effective in treatment IBD patients, i.e. to kill or reprogram the cells (neutrophils, T cells, etc.), rather than just dealing with the cytokines [3].

Some monoclonal antibodies applicable to IBD have been recently developed, such as Ustekinumab, a human monoclonal antibody that blocks both IL-12 and IL-23, and Secukinumab that targets IL-17. In our study, we did find higher protein levels of IL-23 in the patient serum. However, an antibody against IL-23 could be beneficial in patients who have increased IL-23 in their serum. In respect to our findings regarding increased IL- 6 in patients' serum, we also suggested the potential benefit of anti-IL- 6 antibodies as a therapeutic target in IBD [1].

\section{References}

1. Velikova T, Kyurkchiev D, Spassova Z, Karakolev I, Ivanova-Todorova E, et al. (2017) Alterations in cytokine gene expression profile in colon mucosa of Inflammatory Bowel Disease patients on different therapeutic regimens. Cytokine 92: 12-19. [Crossref]

2. Fiocchi C (2014) Integrating Omics: The Future of IBD? Dig Dis 32: 96-102. [Crossref]

3. MacDonald TT (2011) New Cytokine Targets in Inflammatory Bowel Disease. Gastroenterology \& Hepatology 7: 474-476.
Copyright: (C2018 Velikova T. This is an open-access article distributed under the terms of the Creative Commons Attribution License, which permits unrestricted use, distribution, and reproduction in any medium, provided the original author and source are credited.
*Correspondence to: Tsvetelina Velikova, Clinical Immunology, University Hospital Lozenetz, Kozyak 1 str, Sofia 1407, Bulgaria, E-mail: tsvelikova@ medfac.mu-sofia.bg

Received: September 28, 2018; Accepted: October 08, 2018; Published: October 10,2018 